\title{
Improving ontologies by automatic reasoning and evaluation of logical definitions
}

\author{
Sebastian Köhler ${ }^{1,2^{*}}$, Sebastian Bauer ${ }^{1}$, Chris J Mungall ${ }^{3}$, Gabriele Carletti ${ }^{4}$, Cynthia L Smith ${ }^{5}$, Paul Schofield ${ }^{5,6}$, \\ Georgios V Gkoutos ${ }^{7}$ and Peter N Robinson ${ }^{1,2,8^{*}}$
}

\begin{abstract}
Background: Ontologies are widely used to represent knowledge in biomedicine. Systematic approaches for detecting errors and disagreements are needed for large ontologies with hundreds or thousands of terms and semantic relationships. A recent approach of defining terms using logical definitions is now increasingly being adopted as a method for quality control as well as for facilitating interoperability and data integration.

Results: We show how automated reasoning over logical definitions of ontology terms can be used to improve ontology structure. We provide the Java software package GULO (Getting an Understanding of LOgical definitions), which allows fast and easy evaluation for any kind of logically decomposed ontology by generating a composite OWL ontology from appropriate subsets of the referenced ontologies and comparing the inferred relationships with the relationships asserted in the target ontology. As a case study we show how to use GULO to evaluate the logical definitions that have been developed for the Mammalian Phenotype Ontology (MPO).
\end{abstract}

Conclusions: Logical definitions of terms from biomedical ontologies represent an important resource for error and disagreement detection. GULO gives ontology curators a fast and simple tool for validation of their work.

\section{Background}

The steady increase in biomedical data and publications has led to the need for computational methods for integration and analysis [1]. Controlled vocabularies and ontologies for representing biomedical entities, their terms and their relationships are being developed in order to accomplish this task, with the Gene Ontology [2] (GO) probably being the most successful current bio-ontology. There are multiple ontologies for specific disciplines in biomedicine, which enable scientists to deal with the huge amount of data produced, but a major problem is the lack of interoperability between ontologies of different domains of biomedical knowledge.

The Open Biological and Biomedical Ontologies (OBO) Foundry aims to coordinate a family of ontologies that are interoperable and logically well-formed [3]. These ontologies are constantly growing as knowledge grows (e.g., GO currently comprises over 35,000 classes and over 60,000 relationships), which implies that new quality checking

\footnotetext{
* Correspondence: sebastian.koehler@charite.de; peter.robinson@charite.de 'Institute for Medical Genetics and Human Genetics, Charité-

Universitätsmedizin Berlin, Augustenburger Platz 1, 13353 Berlin, Germany Full list of author information is available at the end of the article
}

approaches are needed, since manual creation and maintenance of large ontologies is time-consuming and errorprone.

In principle, a good way to develop ontologies is to define concepts in terms of other more elementary (atomic) concepts (building blocks). Groups involved in the GO [4], the Mammalian Phenotype Ontology (MPO) [5], the Human Phenotype Ontology (HPO) [6-8], and the Worm Phenotype Ontology [9] are now developing logical definitions for ontology terms using terms from other ontologies, with PATO, an ontology of phenotypic qualities, being a key tool in this effort [10-14]. For instance, consider the following logical definition of the HPO term Hypoglycemia, specified in OBO Format:

[Term]

id: HP:0001943 ! Hypoglycemia

intersection_of : PATO:0001163 !

decreased concentration

intersection_of : qualifier PATO:0000460

! abnormal

intersection_of: towards CHEBI:17234! glucose
Ciomed Central

(c) 2011 Köhler et al; licensee BioMed Central Ltd. This is an Open Access article distributed under the terms of the Creative Commons Attribution License (http://creativecommons.org/licenses/by/2.0), which permits unrestricted use, distribution, and reproduction in any medium, provided the original work is properly cited. 
intersection_of: inheres_in FMA:9670! Portion of blood

Hypoglycemia refers to a decreased concentration of glucose in the blood. The logical definition uses relations and follows the pattern described in previous work on defining phenotypes [4]. The logical semantics are made explicit in the translation to the Ontology Web Language (OWL) [15]. The translation used in this manuscript represents a relatively simple design pattern that nonetheless leads to the desired inferences.

\section{Class: Hypoglycemia}

EquivalentTo: 'decreased concentration' and towards some 'glucose.

and inheres_in some 'portion of blood' and qualifier some 'abnormal'

Note that we use the term labels rather than identifiers for the purposes of readability. Here, the class Hypoglycemia is defined as being equivalent to the intersection of all classes of things that are "A concentration which is lower relative to the normal" (decreased concentration), "deviate from the normal or average" (abnormal), with respect to (towards) glucose, and inhering in "blood" (using the term portion of blood from the Foundational Model of Anatomy (FMA) [16]). We use the formal inheres_in relation to relate qualities to their bearers here the bearer of the quality is the blood. The relation towards is used to connect the quality (here, decreased concentration) to the additional entity type on which the quality depends (here glucose) [17]. We use this together with the term for glucose from the Chemical Entities of Biological Interest (ChEBI) ontology [18], essentially stating that the concentration is a concentration "of" glucose. We have thus defined Hypoglycemia as the intersection of these four classes. Defining ontology terms in this way assists in automating ontology construction, and provides a tool for integrative computational analysis of human and model organism phenotypes against the background of the knowledge incorporated in ontologies such as GO, FMA, and ChEBI [14].

In OWL, an ontology is a collection of axioms. An axiom can be thought of as a statement or a sentence, and includes ontological relationships such as those involving is_a or part_of. In the context of this paper, we say that axioms can be asserted (i.e. put there by the ontology curator) or inferred (deduced by a reasoner).

Having created logical definitions, one can apply automatic reasoners, which are systems for computing the logical consequences that can be inferred from a set of asserted axioms. Because reasoning systems can infer the positions of classes in a subsumption hierarchy based on their computable, logical definitions, they can serve as powerful tools in ontology development and maintenance [4]. The asserted subsumption hierarchy of a target ontology (such as the MPO) should be a logical consequence of the definitions of the terms. As seen in Figure 1 the terms abnormal ion homeostasis and abnormal copper homeostasis of the target ontology, the MPO, are defined logically. This has been done by referencing the GO term ion homeostasis for the first and copper homeostasis for the latter MPO term. Since there exists a subsumption axiom between the referenced GO terms (and the PATO terms used are identical) a reasoner will infer that the MPO term abnormal copper homeostasis is a subclass of the MPO term abnormal ion homeostasis.

Thus we assume that this relationship should also be asserted in the MPO, i.e., the knowledge represented in building block ontologies should generally be reflected in the target ontology as well.

We assume that a major goal is to create agreement between the relationships that are asserted in the target ontology and those that can be inferred from the building block ontologies. This can pave the way for extensive data integration with other ontologies to facilitate mining and querying biological knowledge [17]. The creation of the logical definitions for ontology terms mainly depends on manual curation by experts. The curators have to deal with imprecision, missing knowledge and regular changes and updates in the referenced ontologies, and so this can be an enormously complex task. Therefore, one approach to evaluate a target ontology is to run an automatic reasoner over the combined ontologies and logical definitions and then to check how well the manually asserted classlevel axioms agree with the ones found by the reasoner. Depending on the knowledge and the kind of disagreement detected, one may either adapt the logical definitions or revise the subclass relationships of the target ontology. Of course cases may also exist in which the knowledge of the target ontology cannot be fully reasoned over, or cases in which the asserted relationships and inferred relationships represent different views or aspects of knowledge on a particular entity. In many cases, however, it is likely that a disagreement between the asserted hierarchy in ontologies such as the MPO or the HPO with the hierarchies of ontologies for anatomy, biological process, cell types, etc., indicates a logical error that should be manually reviewed by a curator. The developers of the logical definitions therefore need simple tools to evaluate their definitions and the target ontology in a fast and easy way.

Currently, the standard approach is to do minimal assertion in the target ontology, and using a reasoner such as Pellet [19] or HermiT [20] to deductively infer the target ontology polyhierarchy. Most ontology environments such as Protégé4 [21,22] or TopBraid Composer [23] are geared towards this workflow. However, we are faced with the reverse situation in application-driven biological ontologies where the target ontology is often constructed before the building block ontologies, and we want to leverage 


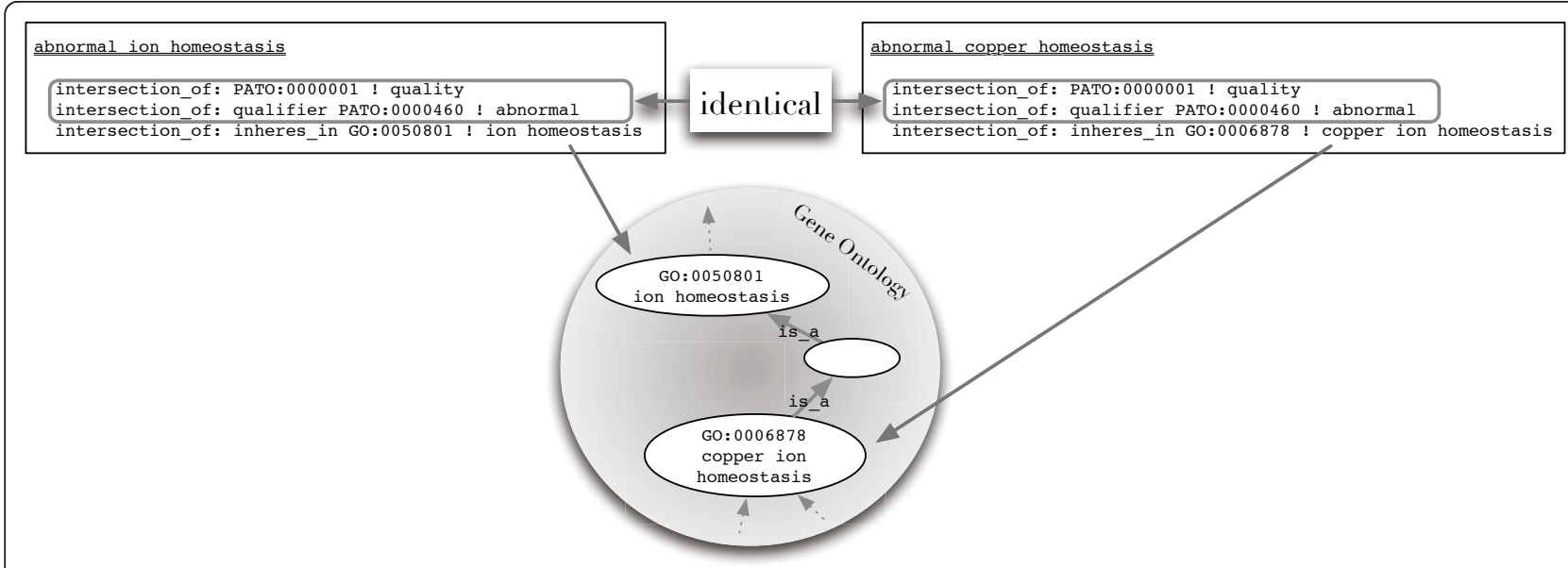

Figure 1 Disagreement of ontologies. In the MPO, abnormal ion homeostasis and abnormal copper homeostasis are not connected by an ancestor/descendent relation. The results of analysis by GULO of logical definitions of the MPO terms suggests that abnormal copper homeostasis should be a subclass of abnormal ion homeostasis, because the term copper ion homeostasis is a descendant of ion homeostasis in the GO. These two terms are used to logically define the corresponding terms in the MPO.

assertions in the target ontology to improve the building block ontologies and the logical definitions through an iterative process of mutual alignment. This has been the case for both the Gene Ontology logical definitions [4] and for phenotypes [17]. For example, the MPO frequently classifies phenotypes anatomically, which when combined with logical definitions allows us to improve anatomical ontologies. Existing OWL-based deductive reasoning environments are less well suited to this "two-way" information flow scenario [24]. In addition, reasoners can have unpredictable performance when used with multiple large ontologies such as the FMA.

Numerous other schemes have been put forward to increase coverage, consistency and quality of biomedical ontologies. These include graph-based approaches [25], linguistic methods for improvement of term names [26], and others (e.g. [27]). A related approach [25] runs only in conjunction with Protégé Frames. The lack of a freely available tool that is based on OBO and OWL semantics and is able to work with more complex logical definitions motivated the work presented here.

\section{Implementation}

In this work, we present and implement a method for using automated reasoning to evaluate a set of logical definitions against the target ontology compared with the knowledge represented by all of the ontologies referenced in the logical definitions. The method first collects only the parts of the referenced ontologies that are relevant for reasoning (Table 1). Note that the referenced ontologies are expected to be provided with subsumption axioms. For the ontologies being considered in this work, it is only necessary to import terms that are directly referenced in the logical definitions and all of their ancestors back to the root in order to infer subclass relationships in the target ontology (Figure 1). We will refer to the graph made up of all referenced terms on an ontology as well as all ancestors on all paths back to the root as the induced ancestral graph of the ontology. Note that we add all relationships between the extracted terms to this graph. For example, by looking at the definitions of the terms from the MPO [28] the induced ancestral graph contains only 1,528 classes of the 35,000 classes in the complete GO (Table 1). Since reasoning does not require any of the other terms in the referenced ontology, one can obtain identical reasoning results over a smaller ontology containing just these terms with a substantial savings in computational resources. There are three types of disagreements between the logical definitions and the target ontology that we would like to detect:

1. A subclass relationship (an is_a link) is implied by the logical definitions but is not explicitly asserted in the target ontology.

2. A subclass relationship (an is_a link) is asserted in the target ontology but is not implied by the logical definitions.

3. The logical definitions imply that two separate classes (terms) in the target ontology are in fact equivalent.

While these three kinds of disagreements are trivial to detect in OWL ontologies, current software such as Protégé was not designed to present lists of detected disagreements in a way that curators can easily use for ontology maintenance. The software presented in this work, GULO (Getting an Understanding of LOgical definitions; Taxonomic note: Gulo gulo, the wolverine, 
Table 110 External ontologies used for the MPO test-run

\begin{tabular}{lll}
\hline Ontology & Number classes in Ontology & Fraction of terms referenced in logical definitions \\
\hline Chemical Entities of Biological Interest & 44,843 & $2.71 \%$ \\
Gene Ontology & 35,090 & $4.35 \%$ \\
Protein Ontology & 26,727 & $0.18 \%$ \\
Molecule Role Ontology & 9,530 & $0.66 \%$ \\
Uber-Anatomy (UBERON) & 8,111 & $17.70 \%$ \\
BRENDA Tissue Ontology & 4,975 & $0.28 \%$ \\
Adult Mouse Anatomical Dictionary & 2,994 & $43.05 \%$ \\
Phenotype, Attribute and Trait Ontology & 2,283 & $23.87 \%$ \\
Cell Ontology & 1,510 & $30.20 \%$ \\
Mouse Pathology & 643 & $18.97 \%$ \\
\hline Total & 140,453 & $7.45 \%$
\end{tabular}

Less than $10 \%$ of the terms of the external ontologies are used by the logical definitions of the MPO terms. "Fraction of terms referenced in logical definitions" denotes the percentage of terms in the ontology that are members of the induced ancestral graph and are used by the logical definitions of the MPO terms.

notably includes owls in its diet), therefore imports the induced ancestral graphs from all ontologies referenced in the logical definitions of the the target ontology, uses computational reasoning to identify the set of all relationships between terms of the target ontology that are implied by the logical definitions and the referred ontologies, and compares them to the relationships that have actually been asserted in the target ontology. Any disagreement is then presented to the user in a set of easyto-use files together with the reasons derived by the reasoner for the disagreement derived by the reasoner. These results can be used by ontology curators for ontology maintenance and debugging.

We provide a stand-alone software implemented in Java that parses a set of definition files (the cross-product logical definitions) and a set of user-defined external ontologies that are referenced in the logical definitions. We will now explain the workflow together with the impact of the several program options that the user can specify. A schematic summary of the workflow is shown in Figure 2.

\section{Generation of relevant ontology subsets and running the reasoner}

All the ontology files (definitions, external ontologies, target ontology) are parsed using the OWL API [29]. Note that for ontologies in $\mathrm{OBO}$ format we use the oboformat library [30], which provides a parser for OBO format 1.4 syntax and an implementation of a mapping to OWL using the OWL API. We also use the OWLTools package [31], a convenience Java API on top of the OWL API, which among other things provides convenient graphoperation capabilities over ontologies.

After parsing the definition file(s) and the external ontologies, a single composite ontology is constructed. By default, the composite ontology is generated using only subsets (the induced ancestral graphs) of the external ontologies; alternatively, the external ontologies are imported in their entirety if the option -s is chosen. The composite ontology (Figure 2: "ontology given to reasoner") is written to file and if desired can be imported into Protégé for manual inspection. Following this, the reasoner is started. The reasoners FaCT++ [32], HermiT [20], or Pellet [19] can be specified using the option $-r$. Note that in order to use $\mathrm{FaCT}++$, the corresponding $\mathrm{FaCT}++$ Java Native Interface library must be defined and available in the Java library path.

\section{Comparison against target ontology}

After reasoning, a new ontology is created from the inferred axioms (Figure 2: "reasoned ontology"). This ontology is then compared against the specified targetontology (-t). GULO performs a comparison in both directions; i.e., the reasoned axioms are checked for presence in the target ontology ("Reasoned ontology $\rightarrow$ Target ontology") and conversely all links between (defined) terms in the target ontology are sought in the reasoned ontology ("Target ontology $\rightarrow$ Reasoned ontology").

\section{Output files}

GULO writes several files to a user-defined output folder (option -o). A list of the most important files that are generated by GULO are given in Figure 2 (Output). The merged ontology and the ontology generated by the reasoner are written to the output folder. During the comparison of these two ontologies the axioms that are found by the reasoner are written to inf_ax_found. txt/inf_ax_not_found.txt according to whether they are present/not present in the specified target ontology. Similarly we report every link in the target ontology between decomposed terms that the reasoner has detected/not detected in the file assert_ax found.txt/assert_ax_not_found.txt

Note that inf_ax_not_found.txt and assert ax_not_found.t $\bar{x}$ t also list the definitions (which give the users hints for debugging) for all the listed 


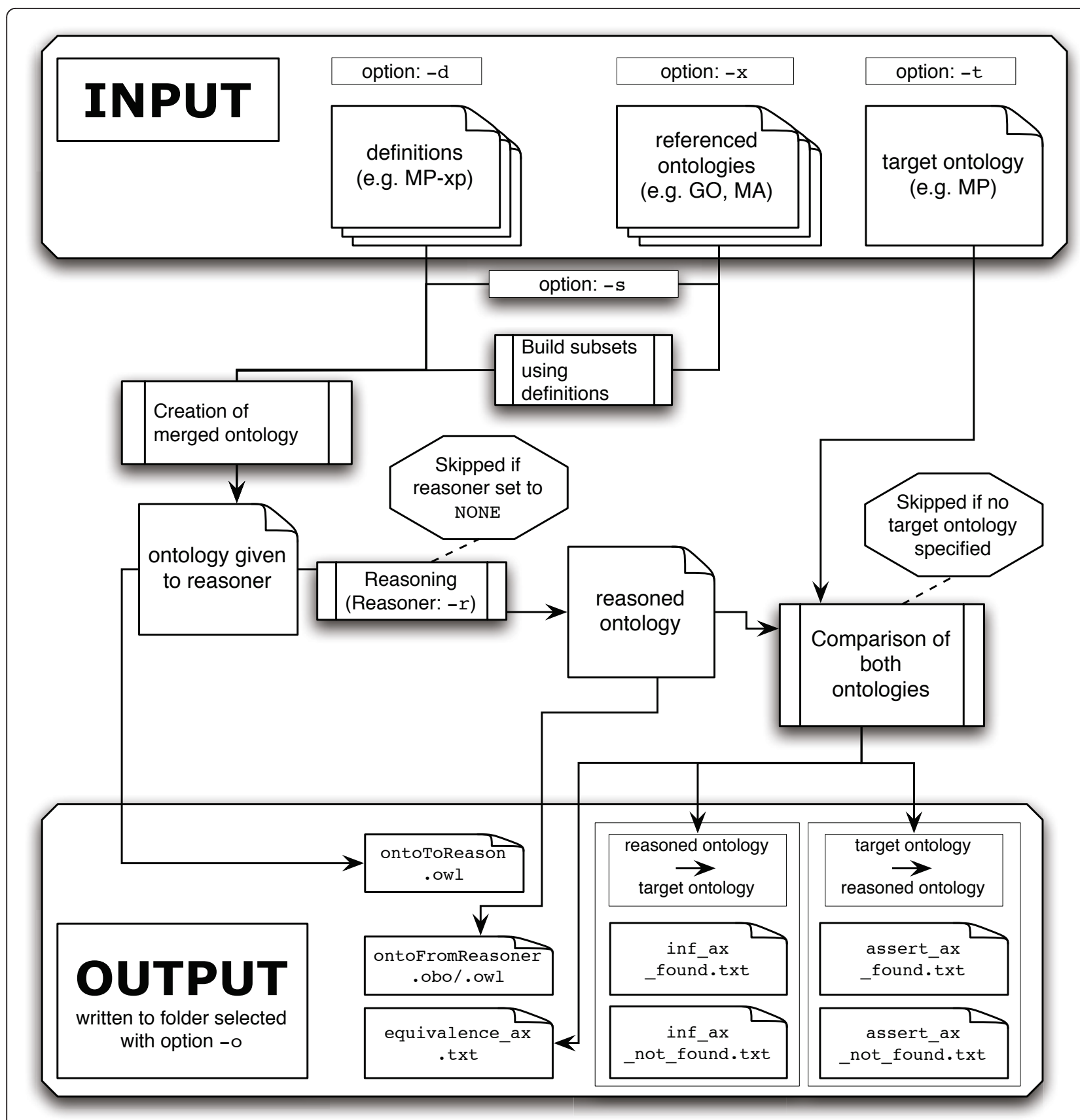

Figure 2 Workflow and options of GULO. A schematic representation of the workflow of GULO and how the user-specified options affect the workflow. Also files read, created and written are depicted.

axioms. Furthermore, a file with inferred equivalence axioms (equivalence_ax.txt) is generated, where the listed equivalences can either point to duplicates in the target ontology (in case of true equivalencies) or to errors in the logical definitions (i.e., when a curator has accidentally used the same definitions for non-equivalent terms).

\section{Results and Discussion}

\section{Software for automated reasoning evaluations}

Here we present an application that automatically reasons over a set of logically decomposed terms and evaluates the resulting ontology against a specified target ontology. The goal of this process is to define terms of a target ontology and use a reasoner to create a new 
ontology by only using the logical definitions. The resulting ontology ideally has the identical structure as the target ontology in which the subclass axioms have been manually asserted. Our program GULO generates subsets of the external ontologies that only contain the terms of the induced ancestral graphs that are referenced in the logical definitions.

Finally, mismatches between the manually asserted subclass axioms and the reasoned axioms are reported and can be used to improve the structure of the target ontology or the axioms used to define the terms contained in the target ontology.

\section{The Mammalian Phenotype Ontology}

To demonstrate that our software is applicable and important for developers and curators of ontologies and/ or logical definitions, we tested it by using the Mammalian Phenotype Ontology (MPO) and the corresponding definition file containing the logical definition of a majority of the MPO terms. Note that for our testing procedure we also generated a bridge between anatomical terms and UBERON [33], since the MPO logical definitions refer to ontologies that are cross-referenced by UBERON (e.g. MA and FMA). A bridging file was generated that transforms the subclass axioms of UBERON (e.g., MA cochlear duct is a subclass of the generic cochlear duct) into correspoding equivalence axioms (e.g., MA coclear duct is defined to be equivalent to the generic cochlear duct in UBERON). This step makes the output of GULO easier to interpret. The code and a runnable jar (GenerateUberonBridge.jar) can be obtained from the GULO subversion repository (see section Availability and requirements). The ontologies used and the output files are also listed there (see folder dataUsed gulopaper/).

\section{Running time and memory}

For testing the computational requirements of our program, we also used the test case of the MPO and the corresponding definitions and ran GULO on a computer with an Intel Core 2 Duo $(2.66 \mathrm{GHz})$ and $4 \mathrm{~GB}$ RAM. When using subset creation (Table 1 ) the reasoning (using HermiT) and evaluation took 115 seconds (run with the VM-option -Xmx500M). Running the same analysis without the construction of subsets gave us identical reasoning/evaluation results, but took around 310 seconds (with the VM option-Xmx1100M).

\section{Evaluating the MPO}

As described above, GULO can be used to detect disagreements or differences in the representation of knowledge between an ontology created from automatically reasoned axioms using logical definitions and a target ontology (MPO), where the axioms between terms have been generated by manual curation (Table 2). The first file presents examples where reasoned axioms
Table 2 GULO results

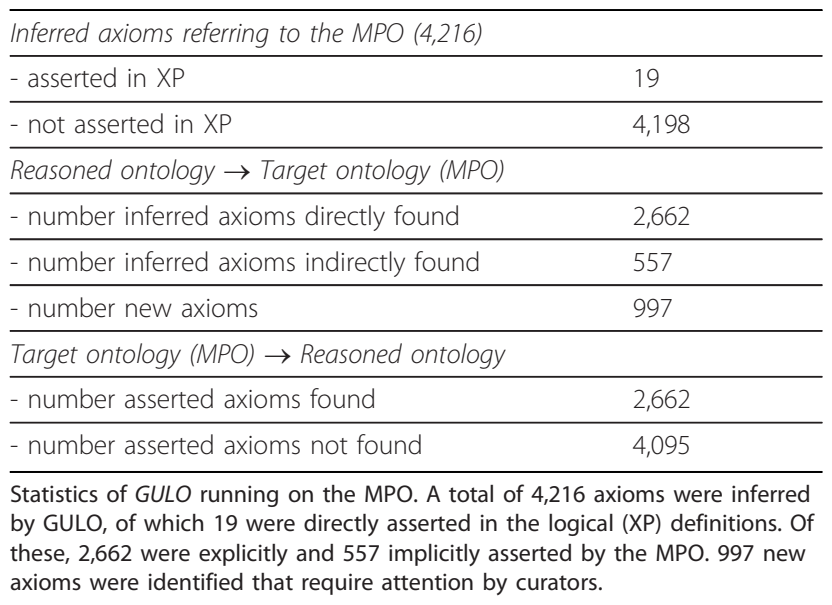

could not be found in the MPO (inf_ax_not_ found.txt).

In the first example the reasoner infers that abnormal copper homeostasis (MP_0003951) is a subclass of abnormal ion homeostasis (MP_0001765). This inference originates from the knowledge representation in the GO, where copper ion homeostasis is a descendant of ion homeostasis (see Figure 1). In the MPO these two terms are currently not in any ancestor-descendant relation, which suggests that further manual curation of these terms is necessary.

A similar example is the reasoned subclass axiom enlarged inguinal lymph nodes (MP_0009623) and enlarged lymph nodes (MP_0000702). As before there is a disagreement between knowledge representation in the MPO and an external ontology (MA). In this case the MA ontology states that inguinal lymph node is a subclass of lymph node, an axiom that is not represented in the MPO. As before we suggest structural changes in the MPO, so that this part of the MPO is aligned with the corresponding part of the MA. Further examples of links suggested by GULO for addition to the MPO are increased prostaglandin level subclass of increased unsaturated fatty acid level, decreased quadriceps weight subclass of decreased muscle weight, and long radius subclass of increased length of long bones.

Note that in total GULO finds 997 subclass axioms between MPO terms that are currently not explicitly asserted in the MPO. These terms and the relationships between them now represent priority items for further manual curation.

\section{Evaluating MPO logical definitions}

Another important feature of GULO is that it can be used to identify curator errors made during the creation of logical definitions. We detected numerous disagreements, but here we will describe only one example. The file equivalence_ax.txt showed us an equivalence 
between abnormal urine uric acid level and abnormal blood uric acid level owing to an erroneous definition of abnormal urine uric acid level which used the MA term for blood instead of the term for urine.

GULO is capable of indicating more complex disagreements. An axiom generated by the reasoner (assert ax_not_found.txt) is the subsumption axiom between abnormal sperm motility (MP_0002674) and abnormal locomotor activity (MP_0001392). This axiom is not contained in the MPO. Both terms were decomposed as abnormal (PATO 0000460 ) and quality (PATO 0000001). The disagreement here comes from the third term used to define both MPO terms, which is in the first case sperm motility (GO_0030317) and locomotion (GO_0040011) for the definition of the latter MPO term. This produces a mixture between a statement about the motility of a single cell (sperm cell) and about the movement behavior of a whole organism (here the mouse). Here we are not confronted with a curator error, rather $\mathrm{GO}$ is too unspecific, as can be seen in the definition of locomotion ("Self-propelled movement of a cell or organism from one location to another"). It is hard to say what the best solution to this problem might be. One solution would be for GO to restructure terms representing movement such that cellular movement and the movement of an entire organism are represented by separate hierarchies of terms.

\section{Conclusions}

We provide a software package (GULO) for automatic reasoning over a set of logical definitions and the ontologies referenced by the definition statements. The referenced ontologies are automatically reduced by removing all classes that are not referred to by the definitions in order to reduce computation time and memory requirements. We assume that the ontology generated by the reasoner optimally should reflect the structure of the manually asserted links given in the target ontology. The reasoned ontology and the target ontology are compared with each other and disagreements are listed. These lists of differences are a powerful resource for the detection of errors in both the logical definition statements and the structure of the target ontology. Of course there may also be cases in which the knowledge of the target ontology cannot be fully reasoned over or cases in which the asserted axioms and inferred axioms represent different views or aspects of knowledge on a particular entity. The methodology presented here can thus be used as a system to help expert curators efficiently identify terms and relationships that require attention. The method is not intended to be used to automatically repair or generate an ontology.

The software presented here uses standard techniques for reasoning over OWL DL ontologies. It is especially designed to be used by curators of biomedical ontologies that use logical, cross-product definitions [4] for the classes of the ontology. This is currently the case for several prominent ontologies in the OBO Language, including the GO, the MPO, the HPO, and the Worm Phenotype Ontology [9]. We have demonstrated the usage of GULO by applying it to the manually created logical definitions of the terms of the MPO. We explained in which way users can get hints for disagreements and errors in both the MPO and the corresponding logical definitions of MPO terms. Curators of logical definitions of any kind of biomedical ontologies can use $G U L O$ as a tool for validation and consistency checking.

\section{Availability and requirements}

- Project name: GULO (Getting an Understanding of LOgical definitions)

- Project home page: http://compbio.charite.de/svn/ hpo/trunk/src/tools/gulo

- Operating system(s): Platform-independent

- Programming language: Java

- Other requirements: Java 1.5 or higher

- License: New BSD License

\section{List of abbreviations}

MP/MPO: Mammalian Phenotype (Ontology); HPO: Human Phenotype Ontology; GO: Gene Ontology; MA: Mouse Anatomy; PATO: Phenotype, Attribute and Trait Ontology

\section{Acknowledgements and Funding}

We would like to thank Michael Ashburner for developing many of the ideas on which this work was built, and for his enthusiastic support. We would also like to thank the two anonymous reviewers for the valuable comments on our manuscript.

This work was supported by grants of the Deutsche

Forschungsgemeinschaft (DFG RO 2005/4-1), the Bundesministerium für Bildung und Forschung (BMBF project number 0313911), and the NIH (R01 HG004838-02).

\section{Author details}

Institute for Medical Genetics and Human Genetics, Charité-

Universitätsmedizin Berlin, Augustenburger Platz 1, 13353 Berlin, Germany. 2Berlin-Brandenburg Center for Regenerative Therapies (BCRT), CharitéUniversitätsmedizin Berlin, Augustenburger Platz 1, 13353 Berlin, Germany. ${ }^{3}$ Lawrence Berkeley National Laboratory, Mail Stop 64R0121, Berkeley, CA 94720, USA. ${ }^{4}$ Dipartimento di Matematica e Informatica, Università di Camerino, Via Madonna delle Carceri 9, 62032 Camerino (MC), Italy. ${ }^{5}$ The Jackson Laboratory, Bar Harbor, ME 04609, USA. ${ }^{6}$ Department of Physiology, Development, and Neuroscience, University of Cambridge, Cambridge, CB2 3EG, UK. ${ }^{7}$ Department of Genetics, University of Cambridge, Downing Street, Cambridge, Cambridge CB2 3EH, UK. ${ }^{8}$ Max Planck Institute for Molecular Genetics, Ihnestrasse 73, 14195 Berlin, Germany.

\section{Authors' contributions}

SK, SB, CJM, PNR, PS, and GVG planned the research work and set up the experiments. SK, SB, and CJM implemented the software. SK, GVG, PS, GC, and CLS performed the evaluations. SK, SB, CJM, and PNR contributed to writing the manuscript. All authors have read and approved the final version of the manuscript.

\section{Competing interests}

The authors declare that they have no competing interests. 
Received: 6 June 2011 Accepted: 27 October 2011

Published: 27 October 2011

\section{References}

1. Robinson PN, Bauer S: Introduction to Bio-Ontologies CRC Press Inc; 2011

2. Ashburner M, Ball C, Blake JA, Botstein D: Gene Ontology: tool for the unification of biology. Nature Genetics 2000

3. Smith B, Ashburner M, Rosse C, Bard J, Bug W, Ceusters W, Goldberg LJ, Eilbeck K, Ireland A, Mungall CJ, Consortium OBI, Leontis N, Rocca-Serra P, Ruttenberg A, Sansone SA, Scheuermann RH, Shah N, Whetzel PL, Lewis SE: The OBO Foundry: coordinated evolution of ontologies to support biomedical data integration. Nat Biotechnol 2007, 25(11):1251-1255.

4. Mungall CJ, Bada M, Berardini TZ, Deegan J, Ireland A, Harris MA, Hill DP Lomax J: Cross-product extensions of the Gene Ontology. J Biomed Inform 2011, 44:80-6.

5. Smith CL, Goldsmith CAW, Eppig JT: The Mammalian Phenotype Ontology as a tool for annotating, analyzing and comparing phenotypic information. Genome Biol 2005, 6:R7.

6. Robinson PN, Köhler S, Bauer S, Seelow D, Horn D, Mundlos S: The Human Phenotype Ontology: a tool for annotating and analyzing human hereditary disease. Am J Hum Genet 2008, 83(5):610-615.

7. Robinson PN, Mundlos S: The human phenotype ontology. Clin Genet 2010, 77(6):525-534.

8. Human Phenotype Ontology. [http://www.human-phenotype-ontology org].

9. Schindelman G, Fernandes JS, Bastiani CA, Yook K, Sternberg PW: Worm Phenotype Ontology: integrating phenotype data within and beyond the $C$. elegans community. BMC Bioinformatics 2011, 12:32.

10. Gkoutos GV, Green ECJ, Mallon AM, Hancock JM, Davidson D: Building mouse phenotype ontologies. Pac Symp Biocomput 2004, 178-189.

11. Gkoutos GV, Green ECJ, Mallon AM, Hancock JM, Davidson D: Using ontologies to describe mouse phenotypes. Genome Biol 2005, 6:R8.

12. Sprague J, Bayraktaroglu L, Bradford Y, Conlin T, Dunn N, Fashena D, Frazer K, Haendel M, Howe DG, Knight J, Mani P, Moxon SAT, Pich C, Ramachandran S, Schaper K, Segerdell E, Shao X, Singer A, Song P, Sprunger B, Slyke CEV, Westerfield M: The Zebrafish Information Network: the zebrafish model organism database provides expanded support for genotypes and phenotypes. Nucleic Acids Res 2008, , 36 Database: D768-D772.

13. Hancock JM, Mallon AM, Beck T, Gkoutos GV, Mungall C, Schofield PN: Mouse, man, and meaning: bridging the semantics of mouse phenotype and human disease. Mamm Genome 2009, 20(8):457-461.

14. Washington NL, Haendel MA, Mungall CJ, Ashburner M, Westerfield M, Lewis SE: Linking human diseases to animal models using ontologybased phenotype annotation. PLoS Biol 2009, 7(11):e1000247.

15. Motik B, Patel-Schneider PF, Parsia B: OWL 2 Web Ontology Language: structural specification and functional-syle syntax 2008 [http://www.w3.org/ TR/owl2-syntax/]

16. Rosse C, Mejino JLV: A reference ontology for biomedical informatics: the Foundational Model of Anatomy. J Biomed Inform 2003, 36(6):478-500.

17. Mungall CJ, Gkoutos GV, Smith CL, Haendel MA, Lewis SE, Ashburner M: Integrating phenotype ontologies across multiple species. Genome Biol 2010, 11:R2.

18. Degtyarenko K, de Matos P, Ennis M, Hastings J, Zbinden M, McNaught A, Alcántara R, Darsow M, Guedj M, Ashburner M: ChEBI: a database and ontology for chemical entities of biological interest. Nucleic Acids Res 2008, , 36 Database: D344-D350.

19. Sirin E, Parsia B, Cuenca Grau B, Kalyanpur A, Katz Y: Pellet: A practical OWL-DL reasoner. Web Semantics 2007, 5(2):51-53.

20. Motik $B$, Shearer $R$, Horrocks I: Optimized reasoning in description logics using hypertableaux. Proc of CADE-21, Volume 4603 of LNCS (LNAI), Heidelberg: Springer 2007, 67-83.

21. Noy NF, Crubezy M, Fergerson RW, Knublauch H, Tu SW, Vendetti J, Musen MA: Protégé-2000: an open-source ontology-development and knowledge-acquisition environment. AMIA Annu Symp Proc 2003, 2003:953.

22. Protégé. [http://protege.stanford.edu].

23. TopQuadrant: TopQuadrant - products - TopBraid Composer. 2011 [http://www.topquadrant.com/products/TB_Composer.html].

24. Bada $M$, Mungall $C$, Hunter $L$ : A call for an abductive reasoning fature in OWL-reasoning tools toward ontology quality control. 5th OWL
Experiences and Directions Workshop (OWLED 2008) 2008 [http://www. webont.org/owled/2008/papers/owled2008eu_submission_44.pdf].

25. Bada $M$, Hunter L: Identification of $\mathrm{OBO}$ nonalignments and its implications for OBO enrichment. Bioinformatics 2008, 24(12):1448-1455.

26. Verspoor K, Dvorkin D, Cohen KB, Hunter L: Ontology quality assurance through analysis of term transformations. Bioinformatics (Oxford, England) 2009, 25(12):i77-84.

27. Ceusters W, Smith B, Kumar A, Dhaen C: Mistakes in medical ontologies: where do they come from and how can they be detected? Stud Health Technol Inform 2004, 102:145-63.

28. Gkoutos GV, Green ECJ, Mallon AM, Hancock JM, Davidson D: Using ontologies to describe mouse phenotypes. Genome Biol 2004, 6:R8.

29. Horridge M, Bechhofer S: The OWL API: A Java API for working with OWL 2 ontologies. 6th OWL Experienced and Directions Workshop, Chantilly, Virginia 2009.

30. oboformat library. [http://code.google.com/p/oboformat/].

31. OWL Tools. [http://code.google.com/p/owltools/].

32. Tsarkov D, Horrocks I: FaCT++ description logic reasoner: system description. Proc of the Int Joint Conf on Automated Reasoning (IJCAR 2006), Volume 4130 of Lecture Notes in Artificial Intelligence, Springer 2006, 292-297.

33. Haendel MA, Gkoutos GG, Lewis SE, Mungall C: Uberon: towards a comprehensive multi-species anatomy ontology. 2009 [http://precedings. nature.com/documents/3592/version/1].

doi:10.1186/1471-2105-12-418

Cite this article as: Köhler et al:: Improving ontologies by automatic reasoning and evaluation of logical definitions. BMC Bioinformatics 2011 12:418.

\section{Submit your next manuscript to BioMed Central and take full advantage of:}

- Convenient online submission

- Thorough peer review

- No space constraints or color figure charges

- Immediate publication on acceptance

- Inclusion in PubMed, CAS, Scopus and Google Scholar

- Research which is freely available for redistribution

Submit your manuscript at www.biomedcentral.com/submit
C) Biomed Central 